\title{
Dynamic Programming Equations for Constrained Stochastic Control
}

\author{
Richard C. Chen \\ Naval Research Laboratory \\ Washington, D.C. 20375 \\ Gilmer L. Blankenship \\ University of Maryland \\ College Park, Maryland 20742
}

\begin{abstract}
In this paper, it is demonstrated that the dynamic programming approach provides a simple and versatile means for analyzing constrained stochastic control problems. Specifically, three such problems are analyzed using this approach. The problems analyzed are the problem of minimizing a discounted cost infnite horizon expectation objective subject to an identically structured constraint, the problem of minimizing a discounted cost infinite horizon minimax objective subject to a discounted expectation constraint, and the problem of minimizing a discounted expected cost objective subject to a minimax constraint. Using a dynamic programming approach, optimality equations are obtained for these problems. The optimality equations derived for the first two problems are apparently novel. Existence and uniqueness of solutions to the dynamic programming equations for the discounted cost infinite horizon problems are explicitly shown using the Banach fixed point theorem. Thus, this paper demonstrates that the dynamic programming theory for unconstrained stochastic control problems can be extended in a direct way to constrained stochastic control problems. The theory developed is illustrated by numerically solving the constrained stochastic control dynamic programming equations derived for simple example problems.
\end{abstract}

\section{Introduction}

Of the number of approaches to studying stochastic control problems, the dynamic programming approach is a classical approach. While this approach has been applied for a number of problems including the riskneutral and risk-sensitive cases, it has not been commonly applied to constrained stochastic control problems. In this paper, the dynamic programming ap- proach is applied to three specific constrained stochastic control problems, thereby demonstrating that constrained control problems can be addressed simply using a dynamic programming approach. The problems analyzed are the problem of minimizing a discounted expectation-objective subject to an identically structured constraint, the problem of minimizing a discounted minimax objective subject to a discounted expectation constraint, and the problem of minimizing a discounted expected cost objective subject to a discounted minimax constraint. Optimality equations are obtained for these problems using a dynamic programming approach, thus demonstrating that the classical dynamic programming theory for unconstrained stochastic control can be extended in a direct way to constrained stochastic control problems.

Chen and Blair [4] study a practically motivated measurement scheduling problem which was a primary motivation for the study of the constrained stochastic control problems of this paper since it is indeed a particular constrained stochastic control problem. Furthermore, in Chen [5], another similar measurement scheduling problem is analyzed using a dynamic programming approach for only the finite horizon case. In particular, a dynamic programming recursion which is analogous to the finite horizon dynamic programming recursions presented in this paper was derived for a measurement scheduling problem in [5]. The results reported here were also derived in [5], in slightly more general terms.

Altman [1] provides a good review of the constrained stochastic control literature. A commonly studied type of constrained stochastic control problem is that which seeks to optimize a classical risk-neutral expectation criterion subject to a constraint on an identically structured risk-neutral expectation. This type of problem was studied for the discounted cost infinite horizon case for example in references [11], [10], [9] and [8]. This paper includes an analysis of this type of problem using a dynamic programming approach. 
It was observed by Coraluppi and Marcus [6] [7] that a certain constrained stochastic control problem provides an alternative approach to risk-sensitive control. This problem is the mixed risk-neutral/minimax control problem in which the risk-neutral expectation objective is minimized subject to a minimax constraint which incorporates the same cost per state-action pair as the expectation objective. This problem is an alternative approach to risk-sensitive control since it also allows the risk of incurring high costs to be adjusted by adjusting the constraint threshold. In this paper, this problem is revisited. In particular, the uniqueness of the solution to the optimality equation for the problem of minimizing a discounted infinite horizon expectation objective subject to a discounted worst-case constraint, which was not explicitly shown in [6] [7], is shown here using the Banach fixed point theorem. Additionally, in this paper, a related constrained stochastic control problem is studied, namely the minimization of the minimax criterion subject to a risk-neutral expectation constraint. This problem also provides yet another alternative approach to risk-sensitive control, as it also allows the risk of incurring high costs to be adjusted. While these two constrained control problems are closely related in this way, the solutions derived here to these problems differ markedly.

In general, the optimal policy for any given problem may be a randomized policy in which the action chosen at a given time depends probabilistically or nondeterministically on some sufficient statistic. For example, for the optimization of a discounted cost infinite horizon expectation criterion subject to a discounted cost infinite horizon expectation constraint, the optimal cost is known to be randomized, and not deterministic [12]. However, in this paper, the space of policies under consideration is restricted to deterministic policies. From a practical point of view, this is justified since in some practical applications, it may not be desirable to implement randomized policies. From a mathematical point of view, restricting the space of policies to deterministic policies results in notation and development which are simpler than those which would be required for randomized policies, for the problems and approach taken in this paper. However, it is conceptually easy to relax this restriction, due to the simplicity of the theory developed. Similarly, while this paper only considers the case of a single constraint for notational convenience, multiple constraints can also be handled easily with the appropriate modifications.

A two-state Markov model representing an error prone system which is to be maintained by machine replacement was presented in [3] and also used in [7]. In this paper, this Markov model is used as the basis of example problems to illustrate the numerical solution of the optimality equations developed.
The following summarizes the contents of this paper and its organization. Mathematical preliminaries including notation and definitions are included subsequently in Section 2. In Section 3, the problem of finding a deterministic policy which optimizes a riskneutral expectation objective subject to an identically structured constraint is addressed using dynamic programming. Both the finite horizon and discounted-cost infinite horizon cases are studied. In Section 4, the problem of finding a deterministic policy which optimizes a minimax (worst-case) objective subject to an expectation constraint is addressed using dynamic programming, for both the finite horizon and discounted cost infinite horizon cases. Additionally, the infinite horizon dynamic programming optimality equation is solved for a two-state machine replacement example. In Section 5, the problem studied in Coraluppi and Marcus [6] [7] is revisited, and the solution of the infinite horizon dynamic programming equation is shown to be unique by applying the Banach fixed point theorem.

\section{Mathematical Preliminaries}

In this section, definitions and notation used in this paper are listed. These are similar to that of [13].

Definition 2.1 A Markov control process is a 5-tuple ( $S, A, q, c, A(\cdot))$ where $S$, the state space, is a Borel space; $A$, the action or control space, is a Borel space; for every $x \in S, A(x) \subset A$ is a nonempty set which represents the set of admissible actions when the system state is $x$; the transition law $q$ is a stochastic kernel on $S$ given $K$, where $K \stackrel{\text { def }}{=}\{(x, u) \mid x \in S, u \in A(x)\}$; and $c: K \rightarrow I R$ is a measurable function.

It is assumed throughout this paper that both $S$ and $A$ are finite sets. Define the space $Z_{t}$ of admissible histories up to time $t$ by $Z_{t}=K \times Z_{t-1}$ for $t \geq 1$ and $Z_{0}=S$. A generic element $h_{t} \in Z_{t}$ is of the form $h_{t}=$ $\left(x_{0}, u_{0}, \ldots, x_{t-1}, u_{t-1}, x_{t}\right)$. Denote by $\mathcal{P}(X)$ the set of all subsets of a Borel space $X$. Also, denote by $B(X)$ the Banach space of real-valued bounded functions $v$ on $X$ with the supremum norm $\|v\|=\sup _{x \in X}|v(x)|$.

\section{Minimization of an Expectation Objective subject to an Expectation Constraint}

In this section, the problem studied is to find a feedback control policy for a given stochastic dynamic system which minimizes a discounted expected cost objective subject to a discounted expectation constraint. Constrained stochastic control problems of this type have been studied extensively [11] [10] [9] [8] [17]. In 
this paper, a direct dynamic programming approach is applied to this type of problem, resulting in dynamic programming equations which characterize the optimal cost. These optimality equations are analogous to those in unconstrained stochastic control theory, and thus, this section generalizes basic dynamic programming theory of unconstrained stochastic control to the case of constrained stochastic control.

\subsection{The Finite Horizon Case}

This section addresses the minimization of an expected cost objective subject to an expectation constraint for the finite horizon case. The definition of this problem requires the following additional notation and assumptions. Assume a Markov control process is given. Assume that $S$ and $A$ are both finite. Let $G$ be the set of all deterministic policies with the property that at each time $N$, the control applied depends on $h_{N}$. That is, $G=\left\{g=\left\{g_{k}\right\} \mid g_{k}: Z_{k} \rightarrow A\right.$ is a function such that $g_{k}\left(h_{k}\right) \in A\left(x_{k}\right)$ for all $h_{k}=$ $\left.\left(x_{0}, u_{0}, \ldots, x_{k-1}, u_{k-1}, x_{k}\right) \in Z_{k}\right\}$.

For any policy $g \in G$ and any $x_{0} \in S$, let $\left\{x_{k} \mid k \geq 1\right\}$ and $\left\{u_{k} \mid k \geq 0\right\}$ be sequences of random variables such that the distribution of $x_{k+1}$ is given by $q\left(\cdot \mid x_{k}, g_{k}\left(x_{0}, u_{0}, \ldots, x_{k-1}, u_{k-1}, x_{k}\right)\right)$ and $u_{k}=g_{k}\left(x_{0}, u_{0}, \ldots, x_{k-1}, u_{k-1}, x_{k}\right)$.

The finite horizon minimization problem with expectation objective and constraint can be formally described as follows. Let $\beta \in I R$ be a given constant such that $\beta \in(0,1]$. Let $\beta_{d} \in I R$ be a given constant such that $\beta_{d} \in(0,1]$. Define the objective function to be minimized as

$$
J_{N}^{g}\left(x_{0}\right)=E\left[\sum_{j=0}^{N-1} \beta^{j} c\left(x_{j}, u_{j}\right)\right]
$$

and the constraint function as

$$
H_{N}^{g}\left(x_{0}\right)=E\left[\sum_{j=0}^{N-1} \beta_{d}^{j} d\left(x_{j}, u_{j}\right)\right] .
$$

Define

$$
\begin{array}{r}
\Phi_{N}(x)=\{\kappa \mid \text { there exists a policy } \\
\left.g \in G \text { such that } H_{N}^{g}(x) \leq \kappa\right\} .
\end{array}
$$

Define for all $x, \kappa$ such that $\kappa \in \Phi_{N}(x)$

$$
J_{N}^{*}(x, \kappa)=\inf _{g \in G} J_{N}^{g}(x)
$$

subject to

$$
H_{N}^{g}(x) \leq \kappa
$$

Let $\gamma \in B(S)$ be a function such that for all $x \in$ $S, \gamma(x) \in \Phi_{N}(x)$. Then the problem to be solved is to find a policy $g_{N, \gamma}^{*} \in G$ such that

$$
J_{N}^{g_{N, \gamma}^{*}}(x)=J_{N}^{*}(x, \gamma(x))
$$

and

$$
H_{N}^{g_{N, \gamma}^{*}}(x) \leq \gamma(x)
$$

The dynamic programming operator for this problem can be defined as follows. Let $F$ be a function such that

$$
F: S \times I R \rightarrow \mathcal{P}(A \times B(S)) .
$$

Define the mapping $T_{F}: B(S \times I R) \rightarrow B(S \times I R)$ by

$$
\begin{aligned}
T_{F}[V](x, \kappa)= & \inf _{u, \gamma^{\prime} \in F(x, \kappa)}\{c(x, u)+ \\
& \left.\beta \sum_{x^{\prime} \in S} q\left(x^{\prime} \mid x, u\right) V\left(x^{\prime}, \gamma^{\prime}\left(x^{\prime}\right)\right)\right\}
\end{aligned}
$$

if $F(x, \kappa) \neq \phi$ and

$$
T_{F}[V](x, \kappa)=\bar{C}
$$

if $F(x, \kappa)=\phi$ where $\bar{C}$ is an arbitrary positive constant. Then the dynamic programming operator is $T_{F_{N}}$ where

$$
\begin{gathered}
F_{N}(x, \kappa)=\left\{u, \gamma^{\prime} \mid u \in A(x) ; \gamma^{\prime}(z) \in \Phi_{N}(z)\right. \text { for all } \\
\left.z \in S ; d(x, u)+\beta_{d} \sum_{y \in S} \gamma^{\prime}(y) q(y \mid x, u) \leq \kappa\right\}
\end{gathered}
$$

The following theorem defines the dynamic programming equation which the optimal cost function satisfies. The proof can be found in [5] and has been omitted here.

Theorem 3.1 Define the sequence of functions $\left\{V_{0}, V_{1}, V_{2}, \ldots\right\}$ by

$$
V_{N+1}(x, \kappa)=T_{F_{N}}\left[V_{N}\right](x, \kappa)
$$

for $N \geq 1, \kappa \in \Phi_{N+1}(x)$ and

$$
V_{0}(x, \kappa)=0
$$

for all $x, \kappa$. For all $x, \kappa, N$ such that $\kappa \in \Phi_{N}(x)$ and $N \geq 1$,

$$
J_{N}^{*}(x, \kappa)=V_{N}(x, \kappa) .
$$

Note that for all $x, \kappa$ such that $\kappa \notin \Phi_{N}(x)$,

$$
V_{N}(x, \kappa)=\bar{C}
$$

\subsection{The Discounted Infinite Horizon Case}

This section addresses the problem of minimization of an expected cost objective with an expectation constraint for the discounted cost infinite horizon case. This problem can be defined as follows. Let the objective discount factor $\beta \in I R$ be a given constant such 
that $0<\beta<1$. Let the constraint discount factor $\beta_{d} \in I R$ be a given constant such that $0<\beta_{d}<1$. Define the objective function to be minimized as

$$
J_{\infty}^{g}\left(x_{0}\right)=E\left[\lim _{N \rightarrow \infty} \sum_{j=0}^{N-1} \beta^{j} c\left(x_{j}, g_{j}\left(h_{j}\right)\right)\right]
$$

and the constraint function as

$$
H_{\infty}^{g}\left(x_{0}\right)=E\left[\lim _{N \rightarrow \infty} \sum_{j=0}^{N-1} \beta_{d}^{j} d\left(x_{j}, g_{j}\left(h_{j}\right)\right)\right] .
$$

Define

$\Phi_{\infty}(x)=\left\{\kappa \mid\right.$ there exists $g \in G$ such that $\left.H_{\infty}^{g}(x) \leq \kappa\right\}$.

Define for all $(x, \kappa)$ such that $\kappa \in \Phi_{\infty}(x)$

$$
J_{\infty}^{*}(x, \kappa)=\inf _{g \in G} J_{\infty}^{g}(x)
$$

subject to

$$
H_{\infty}^{g}(x) \leq \kappa .
$$

Let $\gamma \in B(S)$ be a function such that for all $x \in$ $S, \gamma(x) \in \Phi_{\infty}(x)$. Then the problem to be solved is to find a policy $g_{\gamma}^{*} \in G$ such that

$$
J_{\infty}^{g_{\gamma}^{*}}(x)=J_{\infty}^{*}(x, \gamma(x))
$$

and

$$
H_{\infty}^{g_{\gamma}^{*}}(x) \leq \gamma(x)
$$

The dynamic programming operator is defined as follows. Define

$$
\begin{gathered}
F_{\infty}(x, \kappa)=\left\{u, \gamma^{\prime} \mid u \in A(x) ; \gamma^{\prime}(z) \in \Phi_{\infty}(z)\right. \text { for all } \\
\left.z \in S ; d(x, u)+\beta_{d} \sum_{y \in S} \gamma^{\prime}(y) q(y \mid x, u) \leq \kappa\right\} .
\end{gathered}
$$

Then the dynamic programming operator is $T_{F_{\infty}}$. The following lemma shows existence and uniqueness of the solution to the infinite horizon dynamic programming equation, which will be subsequently defined.

Lemma 3.1 $T_{F_{\infty}}$ is a contraction.

Proof: From the definition of $T$,

$$
\begin{aligned}
& \left\|T_{F} V_{1}-T_{F} V_{2}\right\| \leq \sup _{\{x, \kappa \mid F(x, \kappa) \neq \phi\}} \sup _{\left\{u, \gamma^{\prime} \in F(x, \kappa)\right\}} \\
& \left\{\sum_{x^{\prime} \in S} q\left(x^{\prime} \mid x, u\right) \beta\left|V_{1}\left(x^{\prime}, \gamma^{\prime}\left(x^{\prime}\right)\right)-V_{2}\left(x^{\prime}, \gamma^{\prime}\left(x^{\prime}\right)\right)\right|\right\} \\
& \leq \beta\left\|V_{1}-V_{2}\right\|
\end{aligned}
$$

The following theorem defines the dynamic programming equation which the optimal cost function satisfies. The proof has been omitted here, but can be found in [5].
Theorem 3.2 Let $V_{\infty}$ be the solution to the infinite horizon dynamic programming equation

$$
V_{\infty}=T_{F_{\infty}} V_{\infty}
$$

Then for all $x, \kappa$ such that $\kappa \in \Phi_{\infty}(x)$

$$
J_{\infty}^{*}(x, \kappa)=V_{\infty}(x, \kappa) .
$$

Note that for all $x, \kappa$ such that $\kappa \notin \Phi_{\infty}(x)$,

$$
V_{\infty}(x, \kappa)=\bar{C} \text {. }
$$

\section{Minimization of a Minimax Objective subject to an Expectation Constraint}

In this section, the problem studied is to find a feedback control policy for a given stochastic dynamic system which minimizes a worst-case cost subject to an expectation constraint. Both finite horizon and discounted cost infinite horizon formulations are addressed using a dynamic programming approach. Optimality equations which characterize the optimal cost and policy are obtained.

Risk-neutral stochastic control involves the optimization of the expected value of the cost incurred by the system as it evolves over time. In risk-sensitive control, the expected value as well as the variance and higher-order moments of the cost are taken into consideration by utilizing an exponential function. A risk parameter permits the sensitivity to higher-order moments and thus the sensitivity to risk to be adjusted. Early work in risk-sensitive control was done by Jacobson [14] and Whittle [18], and a review of the area of risk-sensitive control can be found in reference [15]. In McEneaney [15], a certain point regarding the significance of the risk-sensitive control problem is discussed. Specifically, it was noted in [15] that the risksensitive control problem achieves a unification of two certain control methodologies (risk-neutral and robust control).

It was noted in Coraluppi [7] that the risk-sensitive control problem achieves a unification of the risk-neutral methodology and the minimax control methodology, in which the worst-case cost associated with a stochastic control system is minimized [3]. The risk-sensitive control problem achieves this unification since it approaches the minimax control problem as the riskparameter approaches infinity, and the risk-neutral stochastic control problem as the risk-parameter approaches zero. Additionally, [7] notes that by adjusting the risk parameter, the risk-neutral criterion can be improved at the expense of the minimax criterion, or vice versa. 
It was further noted by Coraluppi [7] that the problem of optimizing an expectation objective for a stochastic dynamic system subject to a minimax constraint which has the same cost per state-action pair as the expectation objective provides a means of unifying the risk-neutral and minimax control methodologies in the same way that the risk-sensitive control problem does. Additionally, by adjusting the constraint threshold, one of the two criteria can be improved at the expense of the other.

In this section, a related problem is studied which also has these two properties. In particular, the optimization of the minimax cost for a stochastic dynamic system subject to an expectation constraint is addressed, using a dynamic programming approach. Clearly, for the special case in which the same cost per state-action pair is used in both the minimax objective and the expectation constraint, this problem approaches the riskneutral stochastic control problem as the constraint threshold approaches zero, and approaches the corresponding minimax control problem as the constraint threshold approaches infinity. While this problem is closely related to the optimization of the risk-neutral expected cost subject to a minimax constraint, the solutions derived here to these problems differ markedly.

4.1 Minimization of a Minimax Objective Subject to an Expectation Constraint over a Finite Horizon

This section addresses the minimization of a minimax objective subject to an expectation constraint for the finite horizon case. The development of this section parallels that of Section 3.1. Define the objective function to be minimized as

$$
\begin{aligned}
J_{N}^{g}\left(\bar{x}_{0}\right)= & \left.\sup \bar{x}_{1}^{N-1} \mid P\left[x_{1}^{N-1}=\bar{x}_{1}^{N-1} \mid x_{0}=\bar{x}_{0}\right] \neq 0\right\} \\
& {\left[\sum_{j=0}^{N-1} \beta^{j} c\left(\bar{x}_{j}, u_{j}\right)\right] }
\end{aligned}
$$

and the constraint function as

$$
H_{N}^{g}\left(x_{0}\right)=E\left[\sum_{j=0}^{N-1} \beta_{d}^{j} d\left(x_{j}, u_{j}\right)\right] .
$$

Define

$$
\begin{gathered}
\Phi_{N}(x)=\{\kappa \mid \text { there exists a policy } \\
\left.g \in G \text { such that } H_{N}^{g}(x) \leq \kappa\right\} .
\end{gathered}
$$

Define for all $x, \kappa$ such that $\kappa \in \Phi_{N}(x)$

$$
J_{N}^{*}(x, \kappa)=\inf _{g \in G} J_{N}^{g}(x)
$$

subject to

$$
H_{N}^{g}(x) \leq \kappa
$$

Let $F$ be a function such that

$$
F: S \times I R \rightarrow \mathcal{P}(A \times B(S)) .
$$

Define the mapping $T_{F}: B(S \times I R) \rightarrow B(S \times I R)$ by

$$
\begin{aligned}
T_{F}[V](x, \kappa)= & \inf _{u, \gamma^{\prime} \in F(x, \kappa)}\{c(x, u)+ \\
& \left.\beta \sup _{\left\{x^{\prime} \mid q\left(x^{\prime} \mid x, u\right) \neq 0\right\}} V\left(x^{\prime}, \gamma^{\prime}\left(x^{\prime}\right)\right)\right\}
\end{aligned}
$$

if $F(x, \kappa) \neq \phi$ and

$$
T_{F}[V](x, \kappa)=\bar{C}
$$

if $F(x, \kappa)=\phi$ where $\bar{C}$ is an arbitrary positive constant. The dynamic programming operator is $T_{F_{N}}$ where

$$
\begin{gathered}
F_{N}(x, \kappa)=\left\{u, \gamma^{\prime} \mid u \in A(x) ; \gamma^{\prime}(z) \in \Phi_{N}(z)\right. \text { for all } \\
\left.z \in S ; d(x, u)+\beta_{d} \sum_{y \in S} \gamma^{\prime}(y) q(y \mid x, u) \leq \kappa\right\}
\end{gathered}
$$

The following theorem defines the dynamic programming equation which the optimal cost function satisfies.

Theorem 4.1 Define the sequence of functions $\left\{V_{0}, V_{1}, V_{2}, \ldots\right\}$ by

$$
V_{N+1}(x, \kappa)=T_{F_{N}}\left[V_{N}\right](x, \kappa)
$$

for $N \geq 1, \kappa \in \Phi_{N+1}(x)$ and

$$
V_{0}(x, \kappa)=0
$$

for all $x, \kappa$. For all $x, \kappa, N$ such that $\kappa \in \Phi_{N}(x)$ and $N \geq 1$,

$$
J_{N}^{*}(x, \kappa)=V_{N}(x, \kappa)
$$

4.2 Minimization of a Discounted Minimax Objective Subject to a Discounted Expectation Constraint over an Infinite Horizon

This section addresses the minimization of a minimax objective subject to an expectation constraint for the discounted infinite horizon case. The development of this section parallels that of Section 3.2. The objective function to be minimized is

$$
\begin{aligned}
J_{\infty}^{g}\left(x_{0}\right)= & \sup _{\left\{x_{1}^{\infty} \mid \text { for all } N, P\left[x_{1}^{N} \mid x_{0}\right] \neq 0\right\}} \\
& \left\{\lim _{N \rightarrow \infty} \sum_{j=0}^{N-1} \beta^{j} c\left(x_{j}, g_{j}\left(h_{j}\right)\right)\right\}
\end{aligned}
$$

and the constraint function is

$$
H_{\infty}^{g}\left(x_{0}\right)=E\left[\lim _{N \rightarrow \infty} \sum_{j=0}^{N-1} \beta_{d}^{j} d\left(x_{j}, g_{j}\left(h_{j}\right)\right)\right] .
$$


Define

$\Phi_{\infty}(x)=\left\{\kappa \mid\right.$ there exists $g \in G$ such that $\left.H_{\infty}^{g}(x) \leq \kappa\right\}$.

Define for all $(x, \kappa)$ such that $\kappa \in \Phi_{\infty}(x)$

$$
J_{\infty}^{*}(x, \kappa)=\inf _{g \in G} J_{\infty}^{g}(x)
$$

subject to

$$
H_{\infty}^{g}(x) \leq \kappa
$$

Define

$$
\begin{gathered}
F_{\infty}(x, \kappa)=\left\{u, \gamma^{\prime} \mid u \in A(x) ; \gamma^{\prime}(z) \in \Phi_{\infty}(z)\right. \text { for all } \\
\left.z \in S ; d(x, u)+\beta_{d} \sum_{y \in S} \gamma^{\prime}(y) q(y \mid x, u) \leq \kappa\right\}
\end{gathered}
$$

Lemma 4.1 $T_{F_{\infty}}$ is a contraction

The following theorem defines the dynamic programming equation which the optimal cost function satisfies.

Theorem 4.2 Let $V_{\infty}$ be the solution to the infinite horizon dynamic programming equation

$$
V_{\infty}=T_{F_{\infty}} V_{\infty}
$$

Then for all $x, \kappa$ such that $\kappa \in \Phi_{\infty}(x)$

$$
J_{\infty}^{*}(x, \kappa)=V_{\infty}(x, \kappa) .
$$

\subsection{Illustrative Examples}

In this section, the preceding theory is applied to two example problems which are based on a simple two state Markov process. This Markov process models a system such as a manufacturing or communications system which is subject to failure. The state space is $X=\{0,1\}$, the set of actions is $U=\{0,1\}$, and the transition kernel is denoted by $q$. One state (0) represents the normal working state, while the other state (1) represents a failed or degraded state. The two actions assumed available are a null action (0) which represents doing nothing and a maintenance action (1). If the system is working and nothing is done, then the system fails with some probability of failure. This probability of failure is given by $q(1 \mid 0,0)$. If the system is broken, then it can be maintained by repair or replacement and with a high probability given by $q(0 \mid 1,1)$ the system becomes working. If the system is working, a maintenance action represented by action 1 can be taken, and the system will remain working with probability $q(0 \mid 0,1)$. It is assumed that

$$
q(0 \mid 1,1)=q(0 \mid 0,1)=1 .
$$

Also, it is assumed that

$$
q(0 \mid 0,1)>q(0 \mid 0,0)
$$

That is, it is better to repair the system than to do nothing. If the system is broken and nothing is done, then the system remains broken:

$$
\begin{aligned}
& q(1 \mid 1,0)=1 \\
& q(0 \mid 1,0)=0 .
\end{aligned}
$$

A cost $\bar{c}$ is assumed to be associated with operation in the broken state:

$$
\bar{c}=c(1,0)=c(1,1) .
$$

It is assumed that

$$
c(0,0)=c(0,1)=0 .
$$

Additionally, a cost $\bar{d}$ is assumed to be associated with the repair action:

$$
\bar{d}=d(0,1)=d(1,1) .
$$

It is also assumed that

$$
d(0,0)=d(1,0)=0 .
$$

Two discounted cost infinite horizon problems based on this system are considered. The first problem considered is to minimize the worst-case operation cost subject to a constraint on the expected repair cost. The second problem considered is to minimize the worstcase repair cost subject to a constraint on the expected operation cost.

For the first problem, the minimax objective is given by

$$
\begin{aligned}
J_{\infty}^{g}\left(x_{0}\right)= & \left.\sup x_{1}^{\infty} \mid \text { for all } N, P\left[x_{1}^{N} \mid x_{0}\right] \neq 0\right\} \\
& \left\{\lim _{N \rightarrow \infty} \sum_{j=0}^{N-1} \beta^{j} c\left(x_{j}, g_{j}\left(h_{j}\right)\right)\right\}
\end{aligned}
$$

while the expectation constraint is given by

$$
H_{\infty}^{g}\left(x_{0}\right)=E\left[\lim _{N \rightarrow \infty} \sum_{j=0}^{N-1} \beta_{d}^{j} d\left(x_{j}, g_{j}\left(h_{j}\right)\right)\right] .
$$

Figure (1) - Figure (2) show the optimal cost as a function of constraint threshold for the following parameter values: objective discount $=0.7$, constraint discount $=$ 0.8 , degraded operation cost $=0.4$, repair cost $=0.5$, breakage probability $=0.3$.

The second problem considered is to minimize the worst-case repair cost subject to a constraint on the 
expected operation cost. For this problem, the minimax objective is given by

$$
\begin{aligned}
J_{\infty}^{g}\left(x_{0}\right)= & \sup _{\left\{x_{1}^{\infty} \mid \text { for all } N, P\left[x_{1}^{N} \mid x_{0}\right] \neq 0\right\}} \\
& \left\{\lim _{N \rightarrow \infty} \sum_{j=0}^{N-1} \beta_{d}^{j} d\left(x_{j}, g_{j}\left(h_{j}\right)\right)\right\}
\end{aligned}
$$

while the expectation constraint is given by

$$
H_{\infty}^{g}\left(x_{0}\right)=E\left[\lim _{N \rightarrow \infty} \sum_{j=0}^{N-1} \beta^{j} c\left(x_{j}, g_{j}\left(h_{j}\right)\right)\right] .
$$

Figures (3) and (4) show the optimal cost as a function of constraint threshold for the following parameter values: objective discount $=0.7$, constraint discount $=$ 0.8 , degraded operation cost $=0.4$, repair cost $=0.5$, breakage probability $=0.3$.

\section{Minimization of an Expectation Objective Subject to a Minimax Constraint}

In this section, the mixed risk-neutral/minimax problem which was studied in Coraluppi and Marcus [6] [7] in a partially observed setting is revisited for the fully observed case. Specifically, the uniqueness of the solution to the optimality equation for the discounted infinite horizon mixed risk-neutral/minimax problem, which was not shown explicitly in [6] [7], is shown here using the Banach fixed point theorem.

\subsection{Minimization of an Expectation Objective Subject to a Minimax Constraint over a Finite Horizon}

This section addresses the minimization of an expected value cost with a worst-case constraint for the finite horizon case. The development of this section parallels that of Section 3.1. Define the objective function to be minimized as

$$
J_{N}^{g}\left(x_{0}\right)=E\left[\sum_{j=0}^{N-1} \beta^{j} c\left(x_{j}, u_{j}\right)\right]
$$

and the constraint function as

$$
\begin{aligned}
H_{N}^{g}\left(x_{0}\right)= & \sup _{\left\{\bar{x}_{1}^{N-1} \mid P\left[x_{1}^{N-1}=\bar{x}_{1}^{N-1}\left|x_{0}=\bar{x}_{0}\right| \neq 0\right\}\right.} \\
& {\left[\sum_{j=0}^{N-1} \beta^{j} d\left(\bar{x}_{j}, u_{j}\right)\right] . }
\end{aligned}
$$

Define

$$
\begin{gathered}
\Phi_{N}(x)=\{\kappa \mid \text { there exists a policy } \\
\left.g \in G \text { such that } H_{N}^{g}(x) \leq \kappa\right\} .
\end{gathered}
$$

Define for all $x, \kappa$ such that $\kappa \in \Phi_{N}(x)$

$$
J_{N}^{*}(x, \kappa)=\inf _{g \in G} J_{N}^{g}(x)
$$

subject to

$$
H_{N}^{g}(x) \leq \kappa .
$$

Let $F$ be a function such that

$$
F: S \times I R \rightarrow \mathcal{P}(A)
$$

Define the mapping $T_{F}: B(S \times I R) \rightarrow B(S \times I R)$ by

$$
\begin{gathered}
T_{F}[V](x, \kappa)=\inf _{u \in F(x, \kappa)}\left\{c(x, u)+\beta \sum_{x^{\prime} \in S} q\left(x^{\prime} \mid x, u\right)\right. \\
\left.V\left(x^{\prime},(\kappa-d(x, u)) / \beta\right)\right\}
\end{gathered}
$$

if $F(x, \kappa) \neq \phi$ and

$$
T_{F}[V](x, \kappa)=\bar{C}
$$

if $F(x, \kappa)=\phi$ where $\bar{C}$ is an arbitrary positive constant. Define

$$
\begin{aligned}
F_{N}(x, \kappa)= & \left\{u \mid u \in A(x) ;(\kappa-d(x, u)) / \beta \in \Phi_{N}\left(x^{\prime}\right)\right. \\
& \text { for all } \left.x^{\prime} \in q^{\prime}(x, u)\right\} .
\end{aligned}
$$

The following theorem defines the dynamic programming equation which the optimal cost function satisfies.

Theorem 5.1 Define the sequence of functions $\left\{V_{0}, V_{1}, V_{2}, \ldots\right\}$ by

$$
V_{N+1}(x, \kappa)=T_{F_{N}}\left[V_{N}\right](x, \kappa)
$$

for $N \geq 1, \kappa \in \Phi_{N+1}(x)$ and

$$
V_{0}(x, \kappa)=0
$$

for all $x, \kappa$. For all $x, \kappa, N$ such that $\kappa \in \Phi_{N}(x)$ and $N \geq 1$,

$$
J_{N}^{*}(x, \kappa)=V_{N}(x, \kappa) .
$$

5.2 Minimization of a Discounted Expectation Objective Subject to a Discounted Minimax Constraint over an Infinite Horizon

This section addresses the minimization of an expected value cost with a worst-case constraint for the discounted infinite horizon case. The development of this section parallels that of Section 3.2. The objective function to be minimized is

$$
J_{\infty}^{g}\left(x_{0}\right)=E\left[\lim _{N \rightarrow \infty} \sum_{j=0}^{N-1} \beta^{j} c\left(x_{j}, g_{j}\left(h_{j}\right)\right)\right]
$$


and the constraint function is

$$
\begin{aligned}
H_{\infty}^{g}\left(x_{0}\right)= & \sup _{\left\{x_{1}^{\infty} \mid \text { for all } N, P\left[x_{\mathbf{1}}^{N} \mid x_{0}\right] \neq 0\right\}} \\
& \left\{\lim _{N \rightarrow \infty} \sum_{j=0}^{N-1} \beta_{d}^{j} d\left(x_{j}, g_{j}\left(h_{j}\right)\right)\right\} .
\end{aligned}
$$

Define

$\Phi_{\infty}(x)=\left\{\kappa \mid\right.$ there exists $g \in G$ such that $\left.H_{\infty}^{g}(x) \leq \kappa\right\}$.

Define for all $(x, \kappa)$ such that $\kappa \in \Phi_{\infty}(x)$

$$
J_{\infty}^{*}(x, \kappa)=\inf _{g \in G} J_{\infty}^{g}(x)
$$

subject to

$$
H_{\infty}^{g}(x) \leq \kappa
$$

Define

$$
\begin{aligned}
F_{\infty}(x, \kappa)= & \left\{u \mid u \in A(x) ;(\kappa-d(x, u)) / \beta \in \Phi_{\infty}\left(x^{\prime}\right)\right. \\
& \text { for all } \left.x^{\prime} \in q^{\prime}(x, u)\right\} .
\end{aligned}
$$

Lemma $5.1 T_{F_{\infty}}$ is a contraction

Proof: From the definition of $T$,

$$
\begin{array}{r}
\left\|T_{F} V_{1}-T_{F} V_{2}\right\| \leq \\
\sup _{\{x, \kappa \mid F(x, \kappa) \neq \phi\}} \sup _{\{u \in F(x, \kappa)\}}\left\{\sum_{x^{\prime} \in S} q\left(x^{\prime} \mid x, u\right)\right. \\
\left.\beta\left|V_{1}\left(x^{\prime},(\kappa-d(x, u)) / \beta\right)-V_{2}\left(x^{\prime},(\kappa-d(x, u)) / \beta\right)\right|\right\} \\
\leq \beta\left\|V_{1}-V_{2}\right\| .
\end{array}
$$

The following theorem defines the dynamic programming equation which the optimal cost function satisfies. Note that by the previous lemma, the solution to this equation not only exists but is unique.

Theorem 5.2 Let $V_{\infty}$ be the solution to the infinite horizon dynamic programming equation

$$
V_{\infty}=T_{F_{\infty}} V_{\infty}
$$

Then for all $x, \kappa$ such that $\kappa \in \Phi_{\infty}(x)$

$$
J_{\infty}^{*}(x, \kappa)=V_{\infty}(x, \kappa) .
$$

\section{Conclusion}

In this paper dynamic programming unconstrained stochastic control theory was extended in a direct way to three constrained stochastic control problems. Specifically, using a dynamic programming approach, optimality equations were obtained which characterize the solutions to (1) the problem of minimizing a discounted expectation objective subject to a discounted expectation constraint and (2) the problem of minimizing a discounted minimax objective subject to a discounted expectation constraint and (3) the problem of minimizing a discounted expectation objective subject to a discounted minimax constraint. The second problem appears to be novel to the literature, and is motivated practically as well as theoretically. The dynamic programming equations derived for the first two problems are apparently novel. A feature of the constrained stochastic control problems studied is the restriction of admissible policies to the space of deterministic policies. Also, the Banach fixed point theorem is applied to prove existence and uniqueness of solutions to the dynamic programming equations for the discounted infinite horizon cases. A simple two-state Markov maintenance system which is used in [3] was used here as the basis of example problems for the theory developed.

\section{References}

[1] E. Altman, "Constrained Markov Decision Processes," Research Report, INRIA, No. 2574, May 1995.

[2] R. Bellman and S. Dreyfus, Applied Dynamic Programming, Princeton University Press, 1962.

[3] D. P. Bertsekas, Dynamic Programming and Optimal Control (Volume One), Athena Scientific, 1995.

[4] R. C. Chen and W. D. Blair, "Optimal Measurement Scheduling for Track Accuracy Control for Cued Target Acquisition", Signal and Data Processing of Small Targets 1996, Oliver E. Drummond, Editor, Proc. SPIE 2759, pp. 406-417, 1996.

[5] R. C. Chen, Dynamic Programming Equations for Constrained Stochastic Control, Ph. D. Dissertation, University of Maryland, College Park, Maryland, 2000 .

[6] S. Coraluppi and S. I. Marcus, "Mixed RiskNeutral/Minimax Control of Discrete-Time, FiniteState Markov Decision Processes," IEEE Transactions on Automatic Control Vol. 45, No. 3, March 2000.

[7] S. Coraluppi, Optimal Control of Markov Decision Processes for Performance and Robustness, $\mathrm{Ph}$. D. Dissertation, University of Maryland, College Park, Maryland, 1997.

[8] C. Derman and M. Klein, "Some Remarks on Finite Horizon Markovian Decision Models," Operations Research, 13, pp. 272-278.

[9] E. A. Feinberg and A. Schwartz, "Constrained Dynamic Programming with Two Discount Factors: Applications and an Algorithm," IEEE Transactions on Automatic Control, Vol. 44, No. 3, March 1999, pp. 628 - 631 . 


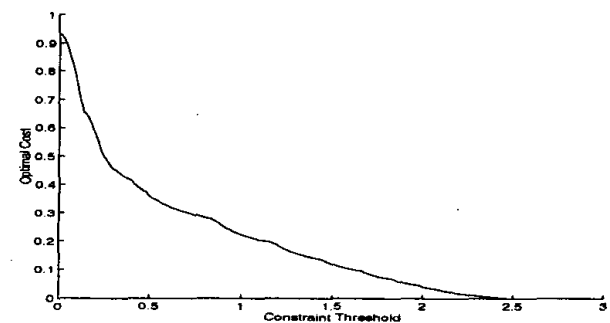

Figure 1: Optimal minimax operating cost subject to expectation repair cost constraint for initially working state with objective discount $=0.7$, constraint discount $=0.8$, degraded operation cost $=0.4$, repair cost $=0.5$, breakage prob. $=$ 0.3

[10] E. A. Feinberg and A. Schwartz, "Constrained Discounted Dynamic Programming," Mathematics of Operations Research, Vol. 21, No. 4, November 1996, pp. $922-945$.

[11] E. A. Feinberg and A. Schwartz, "Constrained Markov Decision Models with Weighted Discounted Rewards," Mathematics of Operations Research, Vol. 20, No. 2, May 1995, pp. 302 - 320.

[12] E. B. Frid, "On Optimal Strategies in Control Problems with Constraints," Theory Prob. Appl., 17, pp. 188 - 192, 1972.

[13] O. Hernandez-Lerma and J. B. Lasserre, Discrete-Time Markov Control Processes: Basic Optimality Criteria, Springer, New York, 1996.

[14] D. H. Jacobson, "Optimal Stochastic Linear Systems with Exponential Performance Criteria and their Relation to Deterministic Differential Games," IEEE Transactions on Automatic Control, AC-18, pp. 124$131,1973$.

[15] W. M. McEneaney, "Risk-Sensitive Control of Nonlinear Systems," SIAG/CST Newsletter, SIAM Activity Group on Control and System Theory, Vol. 4, No. 1, March 1996.

[16] W. M. McEneaney, "Risk-Sensitive Control of Nonlinear Systems," SIAG/CST Newsletter, SIAM Activity Group on Control and System Theory, Vol. 4, No. 2, March 1996.

[17] D. J. White, "Mean, Variance, and Probabilistic Criteria in Finite Markov Decision Processes: A Review", Journal of Optimization Theory and Applications, Vol. 56, No. 1, pp. 1-29, 1988.

[18] P. Whittle, "Risk-Sensitive Linear/Quadratic/Gaussian Control," Advances in Applied Probability, 13, pp. 764-777, 1981.

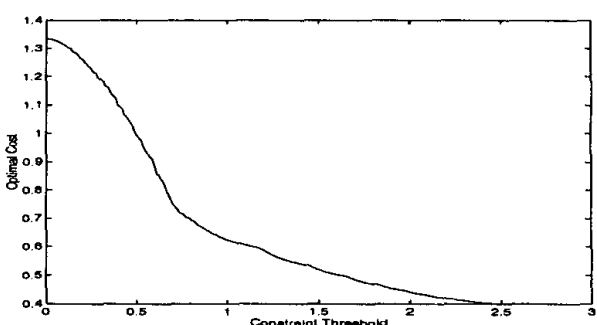

Figure 2: Optimal minimax operating cost subject to expectation repair cost constraint for initially broken state with objective discount $=0.7$, constraint discount $=0.8$, degraded operation cost $=0.4$, repair cost $=0.5$, breakage prob. $=0.3$

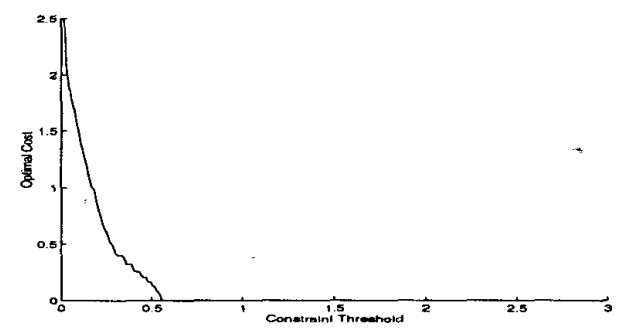

Figure 3: Optimal minimax repair cost subject to expectation operating cost constraint for initially working state with objective discount $=0.7$, constraint discount $=0.8$, degraded operation cost $=0.4$, repair cost $=0.5$, breakage prob. $=$ 0.3

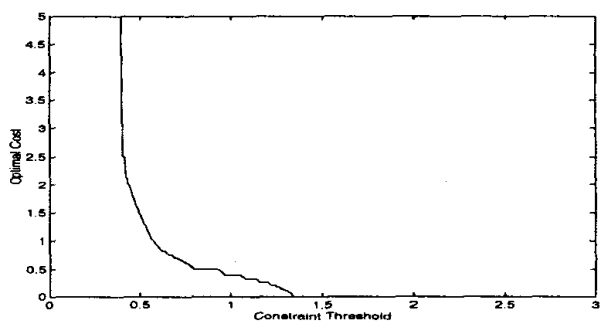

Figure 4: Optimal minimax repair cost subject to expectation operating cost constraint for initially broken state with objective discount $=0.7$, constraint discount $=0.8$, degraded operation cost $=0.4$, repair cost $=0.5$, breakage prob. $=0.3$, 